Applied Mathematical Sciences, Vol. 13, 2019, no. 11, 521 - 531

HIKARI Ltd, www.m-hikari.com

https://doi.org/10.12988/ams.2019.9464

\title{
A Local Stability Theory of Nonsmooth Periodic Orbits: Example II
}

\author{
Pascal Stiefenhofer ${ }^{1}$ \\ Brighton Business School, University of Brighton, UK \\ \& \\ Department of Mathematics, University of Sussex, UK \\ Peter Giesl \\ Department of Mathematics, University of Sussex, UK
}

This article is distributed under the Creative Commons by-nc-nd Attribution License. Copyright (c) 2019 Hikari Ltd.

\begin{abstract}
This paper considers an application of a local theory of exponentially asymptotically stability of nonsmooth periodic orbits derived from a planar dynamical system of autonomous ordinary differential equations with discontinuous right-hand side. Such dynamical systems are encountered in economic modelling in the context of economic growth. In this paper, we revisit an example considered in a companion paper of this journal and show that the explicit solution of the dynamical system is not required in showing exponentially asymptotically stability. We also provide a formula for the basin of attraction. The cost of the new method is also assessed.
\end{abstract}

Keywords: Dynamical Systems, Exponential Asymptotic Stability, Poincaré Map

\section{Introduction}

In complex economic applications of dynamical systems with discontinuous right-hand side the calculation of analytic solutions might be difficult if not

${ }^{1}$ EPSRC Research Grant (Engineering and Physical Science Research Council, 20112016), 1091684, Stability in Nonsmooth Systems with Applications to Biomechanics 
impossible [5], [2], [1], [3], [4]. This is a major drawback in the development of economic models and economic policy analysis, which demand analytic solutions. In this paper, we aim at establishing existence, uniqueness, and exponentially asymptotically stability of a nonsmooth periodic orbit without its explicit calculation. The theory developed in Stiefenhofer and Giesl [7] allows us to do so. We consider an example and compare our method to Poincaré's theory discussed in a companion paper of this journal. Moreover, we characterise the set of stable initial conditions of the periodic orbit, the primary object of economic policy analysis.

We first introduce the model and state the theorem provided in Stiefenhofer and Giesl [7]. Section two discusses how to calculate the smooth conditions of the theorem. Section three shows the calculation of the jump conditions, and section four is a conclusion.

We consider the dynamical system provided in Stiefenhofer and Giesl in a companion paper of the same journal

$$
\dot{x}=f(x),
$$

where $f$ is a discontinuous function at $x_{2}=0$ and $x \in \mathbb{R}^{2}$ such that for $f:=f^{ \pm}$ we have

$$
\dot{x}=f^{ \pm}(x)= \begin{cases}f^{+}(x) & \text { if } x_{2}>0 \\ f^{-}(x) & \text { if } x_{2}<0\end{cases}
$$

Let equation (1) be defined by

$$
\left.\begin{array}{l}
f_{1}^{+}(x)=x_{1}\left(\left(R^{+}\right)^{2}-\left(\left(x_{1}\right)^{2}+\left(x_{2}\right)^{2}\right)\right)-x_{2} \\
f_{2}^{+}(x)=x_{2}\left(\left(R^{+}\right)^{2}-\left(\left(x_{1}\right)^{2}+\left(x_{2}\right)^{2}\right)\right)+x_{1}
\end{array}\right\} \text { if } x_{2}>0
$$

and

$$
\left.\begin{array}{l}
f_{1}^{-}(x)=x_{1}\left(\left(R^{-}\right)^{2}-\left(\left(x_{1}\right)^{2}+\left(x_{2}\right)^{2}\right)\right)-x_{2} \\
f_{2}^{-}(x)=x_{2}\left(\left(R^{-}\right)^{2}-\left(\left(x_{1}\right)^{2}+\left(x_{2}\right)^{2}\right)\right)+x_{1}
\end{array}\right\} \text { if } x_{2}<0
$$

The aim is to calculate the conditions 1-4 of theorem 1:

Theorem 1 (Stiefenhofer and Giesl [7], Theorem $2 p$. 11] Let $\emptyset \neq K \subset \mathbb{R}^{2}$ be a compact, connected and positively invariant set with $f^{ \pm}(x) \neq 0$ for all $x \in K^{ \pm}$. Moreover, assume that $W^{ \pm}: \mathbb{X}^{ \pm} \rightarrow \mathbb{R}^{2}$ are continuous functions and let the orbital derivatives $\left(W^{ \pm}\right)^{\prime}$ exist and be continuous functions in $\mathbb{X}^{ \pm}$and continuously extendable up to $\mathbb{X}_{0}^{ \pm}$. Let following conditions hold:

$$
\begin{aligned}
& \text { 1. } L_{W^{+}(x)}:=\max _{\left\|v^{+}\right\|=e^{-W^{+}(x)}, v^{+} \perp f^{+}(x)} L_{W^{+}}\left(x, v^{+}\right) \leq-\nu<0 \\
& L_{W^{+}}\left(x, v^{+}\right):=e^{2 W^{+}(x)}\left\{\left(v^{+}\right)^{T}\left[D f^{+}(x)\right] v^{+}+\left\langle\nabla W^{+}(x), f^{+}(x)\right\rangle\left\|v^{+}\right\|^{2}\right\} \\
& \text { for all } x \in K^{+} .
\end{aligned}
$$


2. $L_{W^{-}(x)}:=\max _{\left\|v^{-}\right\|=e^{-W^{-}(x)}, v^{-} \perp f^{-}(x)} L_{W^{-}}\left(x, v^{-}\right) \leq-\nu<0$

$L_{W^{-}}\left(x, v^{-}\right):=e^{2 W^{-}(x)}\left\{\left(v^{-}\right)^{T}\left[D f^{-}(x)\right] v^{-}+\left\langle\nabla W^{-}(x), f^{-}(x)\right\rangle\left\|v^{-}\right\|^{2}\right\}$

for all $x \in K^{-}$.

3. $\frac{f_{2}^{-}\left(x_{1}, 0\right)}{f_{2}^{+}\left(x_{1}, 0\right)} \cdot \frac{\sqrt{\left(f_{1}^{+}\left(x_{1}, 0\right)\right)^{2}+\left(f_{2}^{+}\left(x_{1}, 0\right)\right)^{2}}}{\sqrt{\left(f_{1}^{-}\left(x_{1}, 0\right)\right)^{2}+\left(f_{2}^{-}\left(x_{1}, 0\right)\right)^{2}}} e^{W^{-}\left(x_{1}, 0\right)-W^{+}\left(x_{1}, 0\right)}<1$

for all $x \in K^{0}$ with $f_{2}^{+}\left(x_{1}, 0\right)<0, f_{2}^{-}\left(x_{1}, 0\right)<0$.

4. $\frac{f_{2}^{+}\left(x_{1}, 0\right)}{f_{2}^{-}\left(x_{1}, 0\right)} \cdot \frac{\sqrt{\left(f_{1}^{-}\left(x_{1}, 0\right)\right)^{2}+\left(f_{2}^{-}\left(x_{1}, 0\right)\right)^{2}}}{\sqrt{\left(f_{1}^{-}\left(x_{1}, 0\right)\right)^{2}+\left(f_{2}^{-}\left(x_{1}, 0\right)\right)^{2}}} e^{W^{+}\left(x_{1}, 0\right)-W^{-}\left(x_{1}, 0\right)}<1$

for all $x \in K^{0}$ with $f_{2}^{+}\left(x_{1}, 0\right)>0, f_{2}^{-}\left(x_{1}, 0\right)>0$.

Then there is one and only one periodic orbit $\Omega \subset K$. Moreover, $\Omega$ is exponentially asymptotic stable with the real part of the Floquet exponent less or equal $-\nu$ except the trivial one and for its basin of attraction the inclusion $K \subset A(\Omega)$ holds.

\section{The smooth conditions}

From condition one above we have

$$
\begin{aligned}
L_{W^{+}}\left(x, v^{+}\right) & =e^{2 W^{+}(x)}\left\{\left(v^{+}\right)^{T}\left[D f^{+}(x)\right] v^{+}+\left\langle\nabla W^{+}(x), f^{+}(x)\right\rangle\left\|v^{+}\right\|^{2}\right\} \\
& =e^{2 W^{+}(x)}\left[\left(v^{+}\right)^{T} D f^{+}(x) v^{+}+\left(W^{+}(x)\right)^{\prime}\left\|v^{+}\right\|^{2}\right]
\end{aligned}
$$

Hence our aim is to calculate $L_{W^{+}}\left(x, v^{+}\right)<0$ which requires to check that

$$
e^{2 W^{+}(x)}\left[\left(v^{+}\right)^{T} D f^{+}(x) v^{+}+\left(W^{+}(x)\right)^{\prime}\left\|v^{+}\right\|^{2}\right]<0 .
$$

Let $v=\left(\begin{array}{c}-f_{2}^{+} \\ f_{1}^{+}\end{array}\right)$. We first calculate

$\left(v^{+}\right)^{T}\left[D f^{+}\right] v^{+}=\left(\begin{array}{c}-f_{2}^{+} \\ f_{1}^{+}\end{array}\right)^{T}\left(\begin{array}{cc}\left(R^{+}\right)^{2}-r^{2}-2 x_{1}^{2} & -2 x_{1} x_{2}-1 \\ -2 x_{1} x_{2}+1 & \left(R^{+}\right)^{2}-r^{2}-2 x_{2}^{2}\end{array}\right)\left(\begin{array}{c}-f_{2}^{+} \\ f_{1}^{+}\end{array}\right)$

where 


$$
\begin{aligned}
= & \left(\left(R^{+}\right)^{2}-r^{2}-2 x_{1}^{2}\right)\left(f_{2}^{+}\right)^{2}-f_{1}^{+} f_{2}^{+}\left(-4 x_{1} x_{2}\right)+\left(\left(R^{+}\right)^{2}-r^{2}-2 x_{2}^{2}\right)\left(f_{1}^{+}\right)^{2} \\
= & \left(\left(R^{+}\right)^{2}-r^{2}-2 x_{1}^{2}\right)\left(x_{2}^{2}\left(\left(R^{+}\right)^{2}-r^{2}\right)^{2}+x_{1}^{2}+2 x_{1} x_{2}\left(\left(R^{+}\right)^{2}-r^{2}\right)\right) \\
& +\left(\left(R^{+}\right)^{2}-r^{2}-2 x_{2}^{2}\right)\left(x_{1}^{2}\left(\left(R^{+}\right)^{2}-r^{2}\right)^{2}+x_{2}^{2}-2 x_{1} x_{2}\left(\left(R^{+}\right)^{2}-r^{2}\right)\right) \\
& +4 x_{1} x_{2}\left[x_{1} x_{2}\left(\left(R^{+}\right)^{2}-r^{2}\right)^{2}-x_{1} x_{2}+\left(x_{1}^{2}-x_{2}^{2}\right)\left(\left(R^{+}\right)^{2}-r^{2}\right)\right] \\
= & \left(\left(R^{+}\right)^{2}-r^{2}\right)^{3} r^{2}-4 x_{1}^{2} x_{2}^{2}\left(\left(R^{+}\right)^{2}-r^{2}\right)^{2}+\left(\left(R^{+}\right)^{2}-r^{2}\right) r^{2}-2\left(x_{1}^{4}+x_{2}^{4}\right) \\
& -4 x_{1} x_{2}\left[x_{1}^{2}-x_{2}^{2}\right]\left(\left(R^{+}\right)^{2}-r^{2}\right) \\
& 4 x_{1}^{2} x_{2}^{2}\left[\left(\left(R^{+}\right)^{2}-r^{2}\right)^{2}-1\right]+4 x_{1} x_{2}\left(x_{1}^{2}-x_{2}^{2}\right)\left(\left(R^{+}\right)^{2}-r^{2}\right)^{2} \\
= & \left(\left(R^{+}\right)^{2}-r^{2}\right)^{3} r^{2}+\left(\left(R^{+}\right)^{2}-r^{2}\right) r^{2}-4 x_{1}^{2} x_{2}^{2}-2 x_{1}^{4}-2 x_{2}^{4} \\
= & \left(\left(R^{+}\right)^{2}-r^{2}\right)^{3} r^{2}+\left(\left(R^{+}\right)^{2}-r^{2}\right) r^{2}-2\left(x_{1}^{2}+x_{2}^{2}\right)^{2} \\
= & \left(\left(R^{+}\right)^{2}-r^{2}\right)^{3} r^{2}+\left(\left(R^{+}\right)^{2}-r^{2}\right) r^{2}-2 r^{4},
\end{aligned}
$$

which can be rewritten as

$$
v^{T}[D f] v=\left(R^{+}\right)^{6} r^{2}-3\left(R^{+}\right)^{4} r^{4}+3\left(R^{+}\right)^{2} r^{6}-r^{8}+\left(R^{+}\right)^{2} r^{2}-3 r^{4}
$$

The second part of the condition requires to calculate $\left(W^{+}(x)\right)^{\prime}\left\|v^{+}\right\|^{2}$. This in turn requires to define $W$. We start by calculating

$$
\begin{aligned}
\left\|v^{+}\right\|^{2} & =\left(\sqrt{\left(f_{1}^{+}\right)^{2}+\left(-f_{2}^{+}\right)^{2}}\right)^{2} \\
& =r^{2}\left[\left(\left(R^{+}\right)^{2}-r^{2}\right)^{2}+1\right]
\end{aligned}
$$

Next, we define $W^{+}$. Let's assume that $W^{+}=W^{+}(r, \theta)$. Also, our model, given by equations (2) and (3) can be written in polar coordinates as

$$
\begin{aligned}
& \dot{r}^{ \pm}=r^{ \pm}\left(\left(R^{ \pm}\right)^{2}-\left(r^{ \pm}\right)^{2}\right) \\
& \dot{\theta}^{ \pm}=1 .
\end{aligned}
$$

Then the orbital derivative of $W^{+}$is given by

$$
\left(W^{+}\right)^{\prime}=W_{r}^{+} \dot{r}+W_{\theta}^{+} \dot{\theta}=W_{r}^{+} r\left(\left(R^{+}\right)^{2}-r^{2}\right)+W_{\theta}^{+} .
$$

We now define $W$.

$$
\begin{aligned}
& W^{+}(r, \theta)=2\left(-\left(R^{+}\right)^{2} \ln r+\frac{r^{2}}{2}\right)+1.9(\theta+2 \pi) \\
& W^{-}(r, \theta)=1.9 \cdot \theta
\end{aligned}
$$


with

$$
\begin{aligned}
& W_{r}^{+}=\frac{2}{r}\left(\left(-R^{+}\right)^{2}+r^{2}\right) \\
& W_{r}^{-}=0
\end{aligned}
$$

and

$$
\begin{aligned}
& W_{\theta}^{+}=1.9 \\
& W_{\theta}^{-}=1.9
\end{aligned}
$$

thus

$$
\begin{aligned}
& \left(W^{+}(r, \theta)\right)^{\prime}=-2\left(\left(r^{2}-R^{+}\right)^{2}\right)^{2}+1.9 \\
& \left(W^{-}(r, \theta)\right)^{\prime}=1.9
\end{aligned}
$$

We now collect the partial results and state the smooth conditions $L_{W^{+}}\left(x, v^{+}\right)<$ 0

$$
\begin{array}{r}
\left\{\left(R^{+}\right)^{6} r^{2}-3\left(R^{+}\right)^{4} r^{4}+3\left(R^{+}\right)^{2} r^{6}-r^{8}+\left(R^{+}\right)^{2} r^{2}-3 r^{4}+\right. \\
\left.r^{2}\left[\left(\left(R^{+}\right)^{2}-r^{2}\right)^{2}+1\right]\left(-2\left(\left(r^{2}-R^{+}\right)^{2}\right)^{2}+1.9\right)\right\}<0
\end{array}
$$

for $r \in\left[R^{-}, R^{+}\right]$.

and $L_{W^{-}}\left(x, v^{-}\right)<0$

$$
\begin{array}{r}
\left\{\left(R^{-}\right)^{6} r^{2}-3(R)^{4} r^{4}+3\left(R^{-}\right)^{2} r^{6}-r^{8}+\left(R^{-}\right)^{2} r^{2}-3 r^{4}+\right. \\
\left.r^{2}\left[\left(\left(R^{-}\right)^{2}-r^{2}\right)^{2}+1\right] \cdot 1.9\right\}<0
\end{array}
$$

for $r \in\left[R^{-}, R^{+}\right] . K=\left\{r \in \mathbb{R}: R^{-} \leq r \leq R^{+}\right\}$is a positively invariant set.

\section{The jump conditions}

Now, we wan to calculate the jumping conditions a the point $x_{0}=\left(x_{1}, 0\right)$, with $x_{1}<0$ (condition 3 ). Hence, we start by calculating 


$$
\frac{\frac{f_{2}^{-}\left(x_{0}\right)}{\sqrt{\left(f_{1}^{-}\left(x_{0}\right)\right)^{2}+\left(f_{2}^{-}\left(x_{0}\right)\right)^{2}}}}{\frac{f_{2}^{+}\left(x_{0}\right)}{\sqrt{\left(f_{1}^{+}\left(x_{0}\right)\right)^{2}+\left(f_{2}^{+}\left(x_{0}\right)\right)^{2}}}} e^{W^{-}\left(x_{0}\right)-W^{+}\left(x_{0}\right)} .
$$

From equation (2) we have

$$
f_{2}^{+}=x_{2}^{+}\left(\left(R^{+}\right)^{2}-\left(\left(x_{1}^{+}\right)^{2}+\left(x_{2}^{+}\right)^{2}\right)\right)+x_{1}^{+}
$$

and from equation (3) we have

$$
f_{2}^{-}=x_{2}^{-}\left(\left(R^{-}\right)^{2}-\left(\left(x_{1}^{-}\right)^{2}+\left(x_{2}^{-}\right)^{2}\right)\right)+x_{1}^{-} .
$$

We first calculate $\sqrt{\left(f_{1}^{ \pm}\left(x_{0}\right)\right)^{2}+\left(f_{2}^{ \pm}\left(x_{0}\right)\right)^{2}}$. Hence

$$
\begin{aligned}
\left(f_{1}^{ \pm}\left(x_{0}\right)\right)^{2}+\left(f_{2}^{ \pm}\left(x_{0}\right)\right)^{2}= & \left\{x_{1}^{ \pm}\left[\left(R^{ \pm}\right)^{2}-\left(\left(x_{1}^{ \pm}\right)^{2}+\left(x_{2}^{ \pm}\right)^{2}\right)\right]-x_{2}^{ \pm}\right\}^{2} \\
& \times\left\{x_{2}^{ \pm}\left[\left(R^{ \pm}\right)^{2}-\left(\left(x_{1}^{ \pm}\right)^{2}+\left(x_{2}^{ \pm}\right)^{2}\right)\right]+x_{1}^{ \pm}\right\}^{2} .
\end{aligned}
$$

Let $\left.a=\left(\left(x_{1}^{ \pm}\right)^{2}+\left(x_{1}^{ \pm}\right)^{2}\right)-\left(R^{ \pm}\right)^{2}\right)$. Then we have

$$
\begin{aligned}
= & \left((-a) x_{1}^{ \pm}-x_{2}^{ \pm}\right)^{2}+\left((-a) x_{2}^{ \pm}+x_{1}^{ \pm}\right)^{2} \\
= & \left(a x_{1}^{ \pm}\right)^{2}+\left(a x_{2}^{ \pm}\right)^{2}+\left(x_{1}^{ \pm}\right)^{2}+\left(x_{2}^{ \pm}\right)^{2} \\
= & \left\{\left[\left(x_{1}^{ \pm}\right)^{2}+\left(x_{1}^{ \pm}\right)^{2}\right]-\left(R^{ \pm}\right)^{2}\right\}^{2}\left(x_{1}^{ \pm}\right)^{2}+ \\
& \left\{\left[\left(x_{1}^{ \pm}\right)^{2}+\left(x_{1}^{ \pm}\right)^{2}\right]-\left(R^{ \pm}\right)^{2}\right\}^{2}\left(x_{2}^{ \pm}\right)^{2}+\left(x_{1}^{ \pm}\right)^{2}+\left(x_{2}^{ \pm}\right)^{2}
\end{aligned}
$$

which after using $\left(r^{ \pm}\right)^{2}=\left(x_{1}^{ \pm}\right)^{2}+\left(x_{2}^{ \pm}\right)^{2}$ yields

$$
\begin{aligned}
& =\left(\left(r^{ \pm}\right)^{2}-\left(R^{ \pm}\right)^{2}\right)^{2}\left(x_{1}\right)^{2}+\left(\left(r^{ \pm}\right)^{2}-\left(R^{ \pm}\right)^{2}\right)^{2}\left(x_{2}\right)^{2}+\left(r^{ \pm}\right)^{2} \\
& =\left(\left(r^{ \pm}\right)^{2}-\left(R^{ \pm}\right)^{2}\right)^{2}\left(\left(x_{1}\right)^{2}+\left(x_{2}\right)^{2}\right)+\left(r^{ \pm}\right)^{2} \\
& =\left(\left(r^{ \pm}\right)^{2}-\left(R^{ \pm}\right)^{2}\right)^{2}\left(r^{ \pm}\right)^{2}+\left(r^{ \pm}\right)^{2} .
\end{aligned}
$$

Hence, we obtain

$$
\sqrt{\left(f_{1}^{ \pm}\left(x_{0}\right)\right)^{2}+\left(f_{2}^{ \pm}\left(x_{0}\right)\right)^{2}}=\sqrt{\left(\left(r^{ \pm}\right)^{2}-\left(R^{ \pm}\right)^{2}\right)^{2}\left(r^{ \pm}\right)^{2}+\left(r^{ \pm}\right)^{2}} .
$$

We can now calculate the main condition (8) using equations (9), (10), and (11). We obtain

$$
\frac{\frac{f_{2}^{-}\left(x_{0}\right)}{\sqrt{\left(f_{1}^{-}\left(x_{0}\right)\right)^{2}+\left(f_{2}^{-}\left(x_{0}\right)\right)^{2}}}}{\frac{f_{2}^{+}\left(x_{0}\right)}{\sqrt{\left(f_{1}^{+}\left(x_{0}\right)\right)^{2}+\left(f_{2}^{+}\left(x_{0}\right)\right)^{2}}}} e^{W^{-}\left(x_{0}\right)-W^{+}\left(x_{0}\right)}=\frac{\frac{x_{2}^{-}\left(\left(R^{-}\right)^{2}-\left(\left(x_{1}^{-}\right)^{2}+\left(x_{2}^{-}\right)^{2}\right)\right)+x_{1}^{-}}{\sqrt{\left.\left(r^{-}\right)^{2}-\left(R^{-}\right)^{2}\right)^{2}\left(r^{-}\right)^{2}+\left(r^{-}\right)^{2}}}}{\frac{x_{2}^{+}\left(\left(R^{+}\right)^{2}-\left(\left(x_{1}^{+}\right)^{2}+\left(x_{2}^{+}\right)^{2}\right)\right)+x_{1}^{+}}{\sqrt{\left(\left(r^{+}\right)^{2}-\left(R^{+}\right)^{2}\right)^{2}\left(r^{+}\right)^{2}+\left(r^{+}\right)^{2}}}} e^{W^{-}\left(x_{0}\right)-W^{+}\left(x_{0}\right)}
$$


and since $x_{2}^{ \pm}=0$, we obtain

$$
=\frac{\frac{x_{1}^{-}}{\sqrt{\left(\left(r^{-}\right)^{2}-\left(R^{-}\right)^{2}\right)^{2}\left(r^{-}\right)^{2}+1}}}{\frac{x_{1}^{+}}{\sqrt{\left(\left(r^{+}\right)^{2}-\left(R^{+}\right)^{2}\right)^{2}\left(r^{+}\right)^{2}+1}}} e^{W^{-}\left(x_{0}\right)-W^{+}\left(x_{0}\right)}
$$

which since $x_{1}^{+}=x_{1}^{-}$and $r^{+}=r^{-}$yields

$$
=\sqrt{\frac{\left(r^{2}-\left(R^{+}\right)^{2}\right)^{2}+1}{\left(r^{2}-\left(R^{-}\right)^{2}\right)^{2}+1}} e^{W^{-}\left(x_{0}\right)-W^{+}\left(x_{0}\right)} .
$$

We need to check that

$$
\left[\left(r^{2}-\left(R^{+}\right)^{2}\right)^{2}+1\right] e^{2\left(W^{-}\left(x_{0}\right)-W^{+}\left(x_{0}\right)\right)}<\left(r^{2}-\left(R^{-}\right)^{2}\right)^{2}+1 .
$$

Hence we need to show that

$$
W^{-}\left(x_{0}\right)-W^{+}\left(x_{0}\right)<\frac{1}{2} \ln \left(\frac{\left(r^{2}-\left(R^{-}\right)^{2}\right)^{2}+1}{\left(r^{2}-\left(R^{+}\right)^{2}\right)^{2}+1}\right) .
$$

For the jump in the opposite direction we do a similar derivation.

$$
\frac{\frac{f_{2}^{+}\left(x_{0}\right)}{\sqrt{\left(f_{1}^{+}\left(x_{0}\right)\right)^{2}+\left(f_{2}^{+}\left(x_{0}\right)\right)^{2}}}}{\frac{f_{2}^{-}\left(x_{0}\right)}{\sqrt{\left(f_{1}^{-}\left(x_{0}\right)\right)^{2}+\left(f_{2}^{-}\left(x_{0}\right)\right)^{2}}}} e^{W^{+}\left(x_{0}\right)-W^{-}\left(x_{0}\right)}=\frac{\frac{x_{2}^{-}\left(\left(R^{+}\right)^{2}-\left(\left(x_{1}^{-}\right)^{2}+\left(x_{2}^{-}\right)^{2}\right)\right)+x_{1}^{-}}{\sqrt{\left(\left(r^{+}\right)^{2}-\left(R^{+}\right)^{2}\right)^{2}\left(r^{+}\right)^{2}+\left(r^{+}\right)^{2}}}}{\frac{x_{2}^{-}\left(\left(R^{-}\right)^{2}-\left(\left(x_{1}^{-}\right)^{2}+\left(x_{2}^{-}\right)^{2}\right)\right)+x_{1}^{-}}{\sqrt{\left.\left(r^{-}\right)^{2}-\left(R^{+}\right)^{2}\right)^{2}\left(r^{-}\right)^{2}+\left(r^{-}\right)^{2}}}} e^{W^{-}\left(x_{0}\right)-W^{+}\left(x_{0}\right)}
$$

and since $x_{2}^{ \pm}=0, x_{1}^{+}=x_{1}^{-}$and $r^{+}=r^{-}$we obtain

$$
=\sqrt{\frac{\left(r^{2}-\left(R^{-}\right)^{2}\right)^{2}+1}{\left(r^{2}-\left(R^{+}\right)^{2}\right)^{2}+1}} e^{W^{+}\left(x_{0}\right)-W^{-}\left(x_{0}\right)} .
$$

We need to check that

$$
\left[\left(r^{2}-\left(R^{-}\right)^{2}\right)^{2}+1\right] e^{2\left(W^{+}\left(x_{0}\right)-W^{-}\left(x_{0}\right)\right)}<\left(r^{2}-\left(R^{+}\right)^{2}\right)^{2}+1 .
$$

This is equivalent to checking that

$$
W^{+}\left(x_{0}\right)-W^{-}\left(x_{0}\right)<\frac{1}{2} \ln \left(\frac{\left(r^{2}-\left(R^{+}\right)^{2}\right)^{2}+1}{\left(r^{2}-\left(R^{-}\right)^{2}\right)^{2}+1}\right)
$$



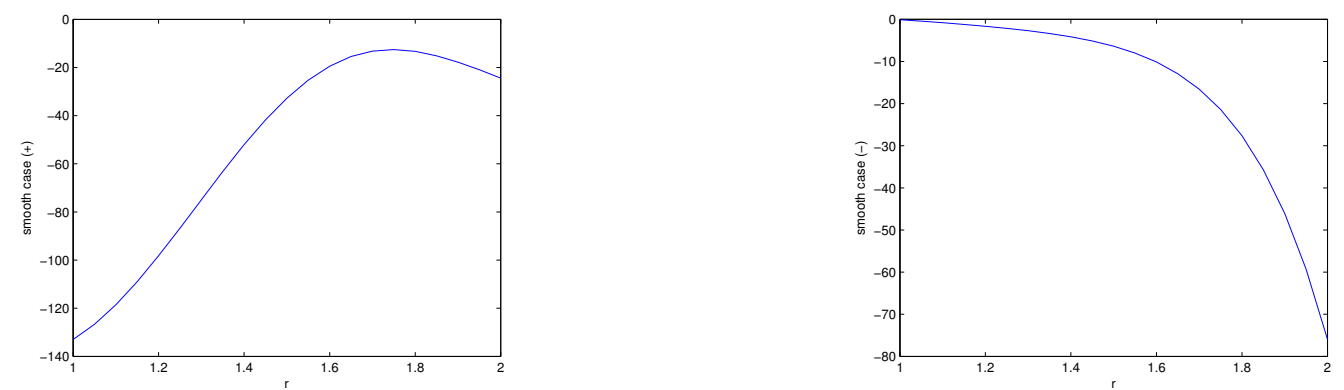

Figure 1: Condition 1: $\operatorname{smooth}(+)$.

Figure 2: Condition 2: $\operatorname{smooth}(-)$.

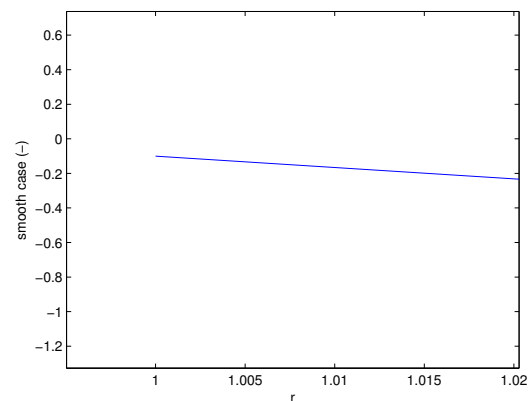

Figure 3: Condition: $\operatorname{smooth}(-)$ at $\mathrm{r}=1$. 
We conclude by collection of conditions (6), (7), (13), and (15) that we need to show that (Condition 1)

$$
\begin{gathered}
\left\{\left(R^{+}\right)^{6} r^{2}-3\left(R^{+}\right)^{4} r^{4}+3\left(R^{+}\right)^{2} r^{6}-r^{8}+\left(R^{+}\right)^{2} r^{2}-3 r^{4}+\right. \\
\left.r^{2}\left[\left(\left(R^{+}\right)^{2}-r^{2}\right)^{2}+1\right]\left(-2\left(\left(r^{2}-R^{+}\right)^{2}\right)^{2}+1.9\right)\right\}<0
\end{gathered}
$$

(Condition 2)

$$
\begin{gathered}
\left\{\left(R^{-}\right)^{6} r^{2}-3(R)^{4} r^{4}+3\left(R^{-}\right)^{2} r^{6}-r^{8}+\left(R^{-}\right)^{2} r^{2}-3 r^{4}+\right. \\
\left.r^{2}\left[\left(\left(R^{-}\right)^{2}-r^{2}\right)^{2}+1\right] \cdot 1.9\right\}<0
\end{gathered}
$$

(Condition 3), where $x_{1}<0, \theta=\pi$ and

$$
\begin{gathered}
W^{-}\left(x_{0}\right)=1.9 \cdot \pi \\
W^{-}\left(x_{0}\right)=2\left(-\left(R^{+}\right)^{2} \ln x_{1}+\frac{\left(x_{1}\right)^{2}}{2}\right)+1.9 \cdot 3 r \\
W^{-}\left(x_{0}\right)-W^{+}\left(x_{0}\right)-\left(\frac{1}{2} \ln \left(\frac{\left(r^{2}-\left(R^{-}\right)^{2}\right)^{2}+1}{\left(r^{2}-\left(R^{+}\right)^{2}\right)^{2}+1}\right)\right)<0
\end{gathered}
$$

(Condition 4), where $x_{1}>0, \theta=2 \pi$ and

$$
\begin{gathered}
W^{-}\left(x_{0}\right)=1.9 \cdot 2 \pi \\
W^{-}\left(x_{0}\right)=2\left(-\left(R^{+}\right)^{2} \ln x_{1}+\frac{\left(x_{1}\right)^{2}}{2}\right)+1.9 \cdot 2 r \\
W^{+}\left(x_{0}\right)-W^{-}\left(x_{0}\right)-\left(\frac{1}{2} \ln \left(\frac{\left(r^{2}-\left(R^{+}\right)^{2}\right)^{2}+1}{\left(r^{2}-\left(R^{-}\right)^{2}\right)^{2}+1}\right)\right)<0
\end{gathered}
$$

which holds for all $r \in\left[R^{-}, R^{+}\right]$with

$$
\begin{aligned}
& R^{-}:=1 \\
& R^{+}:=2
\end{aligned}
$$

by inspection of the graphs, in figure 1, figure 2, figure 3, figure 4, and figure 5 . 


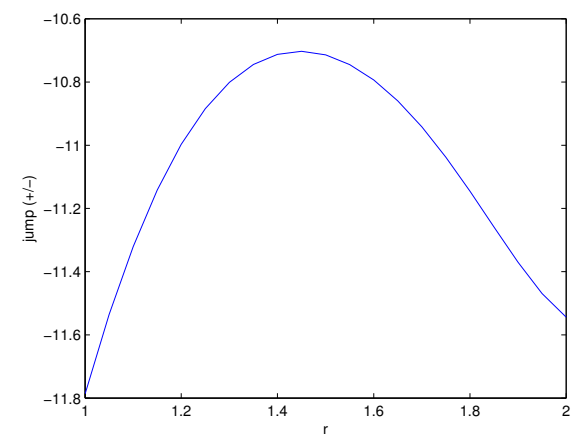

Figure 4: Condition 3: $\operatorname{jump}(+/-)$.

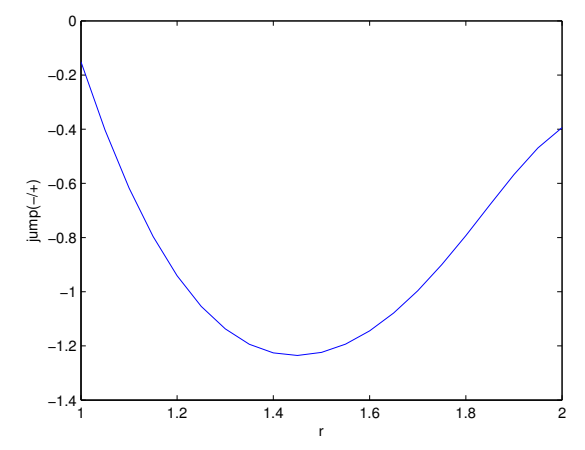

Figure 5: Condition 4: $\operatorname{jump}(-/+)$.

\section{Conclusion}

In a companion paper of this journal we discuss an example of a dynamical system with discontinuous right-hand side (1) defined by equations (2) and (3). We apply classical smooth dynamical systems theory in showing global stability of nonsmooth periodic orbits. Thus we first calculate an explicit solution of a dynamical system defined by a set of ordinary differential equations with discontinuous right-hand side by separation of variables, and then show that this nonsmooth periodic orbit is globally stable by application of a Poincaré map. In this paper, however, we consider an alternative approach to the same example. To show that a periodic orbit is exponentially asymptotically stable the new theory requires to calculate the four conditions of theorem 1 , hence conditions (6), (7), (13), and (15). The theory discussed here does not require the calculation of the explicit solution of the dynamical system in (1). This is the main advantage of our theory. However, the theory considered in this paper requires finding the functions $W^{ \pm}$, which, might not be easy in more complex applications. Yet, there is no general theory available to find such functions. In the case presented here, however, we managed to find such functions and by inspection of the graphs in figure 1 , figure 2 , figure 3 , figure 4 , and figure 5 we conclude that the conditions of the theorem 1 are all satisfied.

\section{References}

[1] J. P. Benassy, A neo-Keynesian model of price and quantity determination in disequilibrium, in: ed. G. Schwödiauer, editor, Equilibrium and disequilibrium in economic theory, Reidel, Dordrecht, 1978, 511-544. https://doi.org/10.1007/978-94-010-1155-6_27

[2] C. Henry, Differential equations with discontinuous right-hand side for palning procedure, Journal of Economic Theory, 4 (1972), 545-551. 
https://doi.org/10.1016/0022-0531(72)90138-x

[3] T. Ito, A Filippov solution of a system of differential equations with discontinuous right-hand side, Economic Letters, 4 (1979), 349-354. https://doi.org/10.1016/0165-1765(79)90183-6

[4] K. G. Löfgren, The corridor and local stability of the effective excess demand hypothesis: A result, Scandinavian Journal of Economics, 81 (1979), 40-47. https://doi.org/10.2307/3439455

[5] F. Mallivaud, The theory of unemployment reconsidered, Basil Blackwell, Oxford, 1977.

[6] P. Stiefenhofer, Stability analysis of non-smooth dynamical systems with an application to biomechanics, University of Sussex, School of Mathematics and Physical Sciences, 2016.

[7] P. Stiefenhofer and P. Giesl, Economic periodic orbits: a theory of asymptotic stability, Nonlinear Analysis and Differential Equations, 7(1) (2019), 9-16. https://doi.org/10.12988/nade.2019.923

Received: April 25, 2019; Published: May 26, 2019 